EUROPEAN ORGANIZATION FOR NUCLEAR RESEARCH

CERN-PPE/92-216

18 Dec 1992

\title{
QCD coherence studies using two particle azimuthal correlations
}

\author{
The OPAL Collaboration
}

\begin{abstract}
From a sample of 146,900 hadronic $Z^{0}$ decays recorded by the OPAL detector at LEP, we have studied the azimuthal correlations of particles in hadronic events. It is expected that these correlations are sensitive to interference effects in QCD. We have compared the data to QCD Monte Carlo models which include and which do not include interference effects. We find that the distributions of azimuthal correlations are not reproduced by the parton shower models we have tested unless interference effects are included, no matter which hadronisation scheme is used.
\end{abstract}

(To be submitted to Z. Phys. C) 


\section{The OPAL Collaboration}

P.D. Acton ${ }^{25}$, G. Alexander ${ }^{23}$, J. Allison ${ }^{16}$, P.P. Allport ${ }^{5}$, K.J. Anderson ${ }^{9}$, S. Arcelli ${ }^{2}$, A. Astbury ${ }^{28}$, D. Axen ${ }^{29}$, G. Azuelos ${ }^{18, a}$, G.A.Bahan ${ }^{16}$, J.T.M.Baines ${ }^{16}$, A.H. Ball ${ }^{17}$, J. Banks ${ }^{16}$, R.J. Barlow ${ }^{16}$, S. Barnett ${ }^{16}$, J.R.Batley ${ }^{5}$, G. Beaudoin ${ }^{18}$, A. Beck ${ }^{23}$, J. Becker ${ }^{10}$, T. Behnke ${ }^{27}$, K.W. Bell ${ }^{20}$, G. Bella ${ }^{23}$, P. Berlich ${ }^{10}$, S. Bethke ${ }^{11}$, O. Biebel ${ }^{3}$, U. Binder ${ }^{10}$, I.J. Bloodworth ${ }^{1}$, P. Bock ${ }^{11}$, B. Boden ${ }^{3}$,

H.M. Bosch ${ }^{11}$, S. Bougerolle ${ }^{29}$, H. Breuker ${ }^{8}$, R.M. Brown ${ }^{20}$, A. Buijs ${ }^{8}$, H.J. Burckhart ${ }^{8}$, C. Burgard ${ }^{27}$, P. Capiluppi ${ }^{2}$, R.K.Carnegie ${ }^{6}$, A.A.Carter ${ }^{13}$, J.R. Carter $^{5}$, C.Y.Chang ${ }^{17}$, D.G.Charlton ${ }^{8}$,

P.E.L. Clarke ${ }^{25}$, I. Cohen ${ }^{23}$, J.C.Clayton1, W.J.Collins ${ }^{5}$, J.E. Conboy ${ }^{15}$, M. Cooper ${ }^{22}$, M. Coupland ${ }^{14}$, M. Cuffiani ${ }^{2}$, S. Dado ${ }^{22}$, G.M.Dallavalle ${ }^{2}$, S. De Jong ${ }^{8}$, L.A. del Pozo ${ }^{5}$, H. Deng ${ }^{17}$, A. Dieckmann ${ }^{11}$, M.Dittmar ${ }^{4}$, M.S.Dixit ${ }^{7}$, E. do Couto e Silva ${ }^{12}$, J.E.Duboscq ${ }^{8}$, E. Duchovni ${ }^{26}$, G. Duckeck ${ }^{11}$, I.P. Duerdoth ${ }^{16}$, D.J.P. Dumas ${ }^{6}$, P.A. Elcombe ${ }^{5}$, P.G. Estabrooks ${ }^{6}$, E. Etzion ${ }^{23}$, H.G. Evans ${ }^{9}$,

F. Fabbri ${ }^{2}$, M.Fincke-Keeler ${ }^{28}$, H.M.Fischer ${ }^{3}$, D.G. Fong ${ }^{17}$, M. Foucher ${ }^{17}$, A. Gaidot ${ }^{21}$, O. Ganel ${ }^{26}$, J.W. Gary ${ }^{4}$, J. Gascon ${ }^{18}$, R.F.McGowan ${ }^{16}$, N.I. Geddes ${ }^{20}$, C. Geich-Gimbel ${ }^{3}$, S.W. Gensler ${ }^{9}$,

F.X. Gentit ${ }^{21}$, G. Giacomelli ${ }^{2}$, V. Gibson ${ }^{5}$, W.R. Gibson ${ }^{13}$, J.D. Gillies ${ }^{20}$, J. Goldberg ${ }^{22}$,

M.J. Goodrick ${ }^{5}$, W. Gorn ${ }^{4}$, C. Grandi ${ }^{2}$, F.C. Grant ${ }^{5}$, J. Hagemann ${ }^{27}$, G.G. Hanson ${ }^{12}$, M. Hansroul ${ }^{8}$, C.K. Hargrove ${ }^{7}$, P.F.Harrison ${ }^{13}$, J. Hart ${ }^{8}$, P.M. Hattersley ${ }^{1}$, M. Hauschild ${ }^{8}$, C.M. Hawkes ${ }^{8}$, E. Heflin ${ }^{4}$,

R.J. Hemingway ${ }^{6}$, R.D. Heuer ${ }^{8}$, J.C. Hill ${ }^{5}$, S.J. Hillier ${ }^{1}$, T. Hilse ${ }^{10}$, D.A. Hinshaw ${ }^{18}$, J.D. Hobbs ${ }^{8}$,

P.R.Hobson ${ }^{25}$, D. Hochman ${ }^{26}$, R.J. Homer ${ }^{1}$, A.K. Honma ${ }^{28, a}$, C.P. Howarth ${ }^{15}$, R.E. Hughes-Jones ${ }^{16}$, R. Humbert ${ }^{10}$, P. Igo-Kemenes ${ }^{11}$, H.Ihssen ${ }^{11}$, D.C.Imrie ${ }^{25}$, A.C. Janissen ${ }^{6}$, A. Jawahery ${ }^{17}$, P.W. Jeffreys ${ }^{20}$, H. Jeremie ${ }^{18}$, M. Jimack ${ }^{2}$, M. Jobes ${ }^{1}$, R.W.L. Jones ${ }^{13}$, P. Jovanovic ${ }^{1}$, C. Juii ${ }^{4}$, D. Karlen ${ }^{6}$, K. Kawagoe ${ }^{24}$, T. Kawamoto ${ }^{24}$, R.K. Keeler ${ }^{28}$, R.G. Kellogg ${ }^{17}$, B.W.Kennedy ${ }^{15}$, S. Kluth ${ }^{5}$, T.Kobayashi ${ }^{24}$, T.P. Kokott ${ }^{3}$, S. Komamiya ${ }^{24}$, L. Köpke', J.F. Kral ${ }^{8}$, R. Kowalewski ${ }^{6}$, J.von $\operatorname{Krogh}^{11}$, J. Krol1 ${ }^{9}$, M. Kuwano ${ }^{24}$, P. Kyberd ${ }^{13}$, G.D. Lafferty ${ }^{16}$, F. Lamarche ${ }^{18}$, J.G. Layter ${ }^{4}$, P. Le Du ${ }^{21}$, P. Leblanc ${ }^{18}$, A.M. Lee ${ }^{17}$, M.H. Lehto ${ }^{15}$, D. Lellouch ${ }^{26}$, P. Lennert ${ }^{11}$, C. Leroy ${ }^{18}$, J. Letts ${ }^{4}$, S. Levegrün ${ }^{3}$, L. Levinson ${ }^{26}$, S.L. Lloyd ${ }^{13}$, F.K. Loebinger ${ }^{16}$, J.M. $\operatorname{Lorah}^{17}$, B. Lorazo $^{18}$, M.J. Losty ${ }^{7}$, X.C. Lou ${ }^{12}$, J. Ludwig ${ }^{10}$, M. Mannelli ${ }^{8}$, S. Marcellini² ${ }^{2}$, G. Maringer ${ }^{3}$, C. Markus ${ }^{3}$, A.J. Martin ${ }^{13}$, J.P. Martin ${ }^{18}$, T. Mashimo ${ }^{24}$, P. Mättig ${ }^{3}$, U. Maur ${ }^{3}$, J. McKenna ${ }^{28}$, T.J. McMahon ${ }^{1}$, J.R. McNutt ${ }^{25}$, F. Meijers ${ }^{8}$, D. Menszner ${ }^{11}$, F.S. Merritt ${ }^{9}$, H. Mes $^{7}$, A. Michelini $^{8}$, R.P. Middleton ${ }^{20}$, G. Mikenberg ${ }^{26}$, J. Mildenberger ${ }^{6}$, D.J. Miller ${ }^{15}$, R. Mir ${ }^{12}$, W. Mohr ${ }^{10}$, C. Moisan ${ }^{18}$, A. Montanari², T. Mori ${ }^{24}$, M. Morii ${ }^{24}$, T. Mouthuy ${ }^{12, b}$, B. Nellen ${ }^{3}$, H.H. Nguyen ${ }^{9}$, M. Nozaki ${ }^{24}$, S.W.O'Neale ${ }^{8, c}$, F.G.Oakham ${ }^{7}$, F. Odorici ${ }^{2}$, H.O. Ogren ${ }^{12}$, C.J. Oram ${ }^{28, a}$, M.J. Oreglia ${ }^{9}$, S. Orito ${ }^{24}$, J.P.Pansart ${ }^{21}$,

B. Panzer-Steindel ${ }^{8}$, P. Paschievici ${ }^{26}$, G.N.Patrick ${ }^{20}$, N.Paz-Jaoshvili ${ }^{23}$, P. Pfister ${ }^{10}$, J.E. Pilcher ${ }^{9}$, D. Pitman ${ }^{28}$, D.E.Plane ${ }^{8}$, P. Poffenberger ${ }^{28}$, B. Poli ${ }^{2}$, A. Pouladdej ${ }^{6}$, E. Prebys ${ }^{8}$, T.W.Pritchard ${ }^{13}$, H. Przysiezniak ${ }^{18}$, G. Quast ${ }^{27}$, M.W. Redmond ${ }^{9}$, D.L. Rees ${ }^{1}$, G.E. Richards ${ }^{16}$, D. Robinson ${ }^{8}$, A. Rollnik ${ }^{3}$, J.M. Roney ${ }^{9}$, E. Ros $^{8}$, S. Rossberg ${ }^{10}$, A.M. Rossi ${ }^{2, d}$, M. Rosvick ${ }^{28}$, P. Routenburg ${ }^{6}$, K. Runge ${ }^{10}$, O.Runolfsson ${ }^{8}$, D.R. Rust ${ }^{12}$, M.Sasaki ${ }^{24}$, C.Sbarra ${ }^{8}$, A.D. Schaile ${ }^{10}$, O. Schaile ${ }^{10}$, W.Schappert ${ }^{6}$, P.Scharff-Hansen ${ }^{8}$, P.Schenk ${ }^{28}$, H. von der Schmitt ${ }^{11}$, S. Schreiber ${ }^{3}$, C.Schwick ${ }^{27}$, J.Schwiening ${ }^{3}$, W.G.Scott ${ }^{20}$, M.Settles ${ }^{12}$, T.G.Shears ${ }^{5}$, B.C.Shen ${ }^{4}$, C.H.Shepherd-Themistocleous ${ }^{7}$, P.Sherwood ${ }^{15}$, R.Shypit ${ }^{29}$, A.Simon ${ }^{3}$, P.Singh ${ }^{13}$, G.P.Siroli ${ }^{2}$, A.Skuja ${ }^{17}$, A.M.Smith ${ }^{8}$, T.J.Smith ${ }^{8}$,

G.A.Snow ${ }^{17}$, R. Sobie ${ }^{28, e}$, R.W.Springer ${ }^{17}$, M.Sproston ${ }^{20}$, K. Stephens ${ }^{16}$, J.Steuerer ${ }^{28}$,

R.Ströhmer ${ }^{11}$, D.Strom ${ }^{30}$, T. Takeshita ${ }^{24, f}$, P. Taras $^{18}$, S. Tarem ${ }^{26}$, M. Tecchio ${ }^{9}$, P.Teixeira-Dias ${ }^{11}$,

N. Tesch ${ }^{3}$, N.J. Thackray ${ }^{1}$, G. Transtromer ${ }^{25}$, N.J. Tresilian ${ }^{16}$, T. Tsukamoto ${ }^{24}$, M.F.Turner ${ }^{5}$, G. Tysarczyk-Niemeyer ${ }^{11}$, D. Van den plas $^{18}$, R. Van Kooten ${ }^{8}$, G.J. VanDalen ${ }^{4}$, G. Vasseur ${ }^{21}$, C.J. Virtue ${ }^{7}$, A.Wagner ${ }^{27}$, D.L. Wagner ${ }^{9}$, C. Wah ${ }^{10}$, J.P. Walker ${ }^{1}$, C.P. Ward ${ }^{5}$, D.R. Ward ${ }^{5}$, P.M.Watkins ${ }^{1}$, A.T.Watson ${ }^{1}$, N.K. Watson ${ }^{8}$, M. Weber ${ }^{11}$, P. Weber ${ }^{6}$, S. Weisz ${ }^{8}$, P.S. Wells ${ }^{8}$,

N. Wermes ${ }^{11}$, M.A. Whalley ${ }^{1}$, G.W.Wilson ${ }^{4}$, J.A. Wilson ${ }^{1}$, V-H. Winterer ${ }^{10}$, T. Wlodek ${ }^{26}$,

S. Wotton ${ }^{11}$, T.R. Wyatt ${ }^{16}$, R. Yaari ${ }^{26}$, A. Yeaman ${ }^{13}$, G. Yekutieli ${ }^{26}$, M. Yurko $^{18}$, W. Zeuner ${ }^{8}$, G.T.Zorn ${ }^{17}$. 
${ }^{1}$ School of Physics and Space Research, University of Birmingham, Birmingham, B15 2TT, UK

${ }^{2}$ Dipartimento di Fisica dell' Università di Bologna and INFN, Bologna, 40126, Italy

${ }^{3}$ Physikalisches Institut, Universität Bonn, D-5300 Bonn 1, FRG

${ }^{4}$ Department of Physics, University of California, Riverside, CA 92521 USA

${ }^{5}$ Cavendish Laboratory, Cambridge, CB3 0HE, UK

${ }^{6}$ Carleton University, Dept of Physics, Colonel By Drive, Ottawa, Ontario K1S 5B6, Canada

${ }^{7}$ Centre for Research in Particle Physics, Carleton University, Ottawa, Ontario K1S 5B6, Canada

${ }^{8} \mathrm{CERN}$, European Organisation for Particle Physics, 1211 Geneva 23, Switzerland

${ }^{9}$ Enrico Fermi Institute and Department of Physics, University of Chicago, Chicago Illinois 60637, USA

${ }^{10}$ Fakultät für Physik, Albert Ludwigs Universität, D-7800 Freiburg, FRG

${ }^{11}$ Physikalisches Institut, Universität Heidelberg, Heidelberg, FRG

${ }^{12}$ Indiana University, Dept of Physics, Swain Hall West 117, Bloomington, Indiana 47405, USA

${ }^{13}$ Queen Mary and Westfield College, University of London, London, E1 4NS, UK

${ }^{14}$ Birkbeck College, London, WC1E 7HV, UK

${ }^{15}$ University College London, London, WC1E 6BT, UK

${ }^{16}$ Department of Physics, Schuster Laboratory, The University, Manchester, M13 9PL, UK

${ }^{17}$ Department of Physics and Astronomy, University of Maryland, College Park, Maryland 20742, USA

${ }^{18}$ Laboratoire de Physique Nucléaire, Université de Montréal, Montréal, Quebec, H3C 3J7, Canada

${ }^{20}$ Rutherford Appleton Laboratory, Chilton, Didcot, Oxfordshire, OX11 0QX, UK

${ }^{21}$ DAPNIA/SPP, CEN Saclay, F-91191 Gif-sur-Yvette, France

${ }^{22}$ Department of Physics, Technion-Israel Institute of Technology, Haifa 32000, Israel

${ }^{23}$ Department of Physics and Astronomy, Tel Aviv University, Tel Aviv 69978, Israel

${ }^{24}$ International Centre for Elementary Particle Physics and Dept of Physics, University of Tokyo,

Tokyo 113, and Kobe University, Kobe 657, Japan

${ }^{25}$ Brunel University, Uxbridge, Middlesex, UB8 3PH UK

${ }^{26}$ Nuclear Physics Department, Weizmann Institute of Science, Rehovot, 76100, Israel

${ }^{27}$ Universität Hamburg/DESY, II Inst für Experimental Physik, 2000 Hamburg 52, Germany

${ }^{28}$ University of Victoria, Dept of Physics, P O Box 3055, Victoria BC V8W 3P6, Canada

${ }^{29}$ University of British Columbia, Dept of Physics, 6224 Agriculture Road, Vancouver BC V6T 1Z1, Canada

${ }^{30}$ University of Oregon, Dept of Physics, Eugene, Oregon 97403, USA

${ }^{a}$ Also at TRIUMF, Vancouver, Canada V6T $2 \mathrm{~A} 3$

${ }^{b}$ Now at Centre de Physique des Particules de Marseille, Faculté des Sciences de Luminy, Marseille ${ }^{c}$ On leave from Birmingham University, Birmingham B15 2TT, UK

${ }^{d}$ Now at Dipartimento di Fisica, Università della Calabria and INFN, 87036 Rende, Italy

${ }^{e}$ And IPP, McGill University, High Energy Physics Department, 3600 University Str, Montreal, Quebec H3A 2T8, Canada

${ }^{f}$ Also at Shinshu University, Matsumoto 390, Japan 


\section{Introduction}

A complete calculation of multigluon emission has to take into account the interference amongst all the amplitudes [1]. This is the so-called coherence phenomenon in QCD. In $\mathrm{e}^{+} \mathrm{e}^{-}$annihilation into hadrons, interference effects provide an explanation of the shape of the momentum spectra of particles, and their evolution with energy [2]. They also give a possible explanation of the "string effect" observed in three-jet hadronic events [3, 4]. The majority of multihadronic events are two-jet like because the emitted gluons are mostly either collinear with the quarks and/or have low energy with respect to the energy scale of the reaction (soft gluons). Coherence effects are also predicted for this class of events and will be investigated through a study of angular correlations between particles as discussed below.

The $\mathrm{e}^{+} \mathrm{e}^{-} \rightarrow q \bar{q} g g$ cross section when one gluon is soft can be factorized as $[5,6]$

$$
\sigma\left(q_{1} \bar{q}_{2} g_{3} g_{4}\right)=\sigma\left(q_{1} \bar{q}_{2} g_{3}\right) \sigma\left(q_{1} \bar{q}_{2} g_{4}\right) C / \sigma\left(q_{1} \bar{q}_{2}\right)
$$

where $\mathrm{C}$ is a correlation term given by

$$
C=1+\frac{N^{2}}{N^{2}-1}\left(\frac{\xi_{13} \xi_{24}+\xi_{14} \xi_{23}}{\xi_{12} \xi_{34}}-1\right)
$$

where $\mathrm{N}$ is the number of colours, $\xi_{i j}=1-\cos \theta_{i j}\left(\theta_{i j}\right.$ is the angle between partons $\mathrm{i}$ and $\left.\mathrm{j}\right)$.

When both $g_{3}$ and $g_{4}$ are soft, the $q$ and the $\bar{q}$ are nearly collinear and define a natural axis with respect to which one can define the pseudorapidity of the two gluons, $\eta_{3}$ and $\eta_{4}$, respectively, $\eta_{34}\left(=\eta_{3}-\eta_{4}\right)$ and the relative azimuth $\varphi_{34}$. The pseudorapidity is $\eta=-\ln \tan (\theta / 2)$, where $\theta$ is the angle between the particle and the axis. In this case, $\mathrm{C}$ reduces to

$$
C=1+\frac{N^{2}}{N^{2}-1}\left(\frac{\cos \varphi_{34}}{\cosh \eta_{34}-\cos \varphi_{34}}\right) \text {. }
$$

When $\varphi_{34} \rightarrow \pi$, the correlation term becomes smaller than 1 , so that the emission of gluons opposite in azimuth to $g_{3}$ is suppressed. The effect is maximal when $\eta_{34} \approx 0$, for which $\mathrm{C}(\pi) / \mathrm{C}(\pi / 2)=7 / 16$. This suppression has the same dynamical origin as the string effect. Assuming Local Parton Hadron Duality [7], such a suppression is expected at the hadron level.

From the expression for the $q \bar{q} g g$ cross section given above and from the fact that the correlation term depends on the pseudorapidities and azimuth of the emitted gluons (equation 2), it follows that the interference effects can be studied by looking at azimuthal correlations between particles (partons or hadrons) using the expression:

$$
\frac{d C}{d \varphi}\left(\eta_{1}, \eta_{2}, \varphi\right)=\sigma \frac{d \sigma}{d \eta_{1} d \eta_{2} d \varphi} /\left(\frac{d \sigma}{d \eta_{1}} \cdot \frac{d \sigma}{d \eta_{2}}\right)
$$

where $\eta_{i}$ is the pseudorapidity of particles with respect to some axis and $\varphi$ is their relative azimuth. From now on, the subscripts 1 and 2 refer to the two particles of any pair.

In this paper, azimuthal correlations are studied in multihadronic events produced in $\mathrm{Z}^{0}$ decays at LEP, using two methods:

- The first method uses the Energy-Multiplicity-Multiplicity Correlations (EMMC) proposed by Dokshitzer, Khoze, Marchesini and Webber [6], which avoid the selection of two-jet events and the definition of an event axis. Each particle of an event defines in turn an axis with respect to which one calculates the pseudorapidities and the relative azimuth of any pair of the other 
particles. Both particles of the pair are required to belong to the same pseudorapidity interval. In order to reflect the direction of the initial quarks, each correlation defined by (3) is weighted by the energy of the particle defining the reference axis. At leading order the EMMC are given by the correlation function (2). Higher order corrections have been recently calculated and are found to be important [8]. The correlation at $\varphi=\pi$ is predicted to be $\approx 0.93$, but in [8], it is argued that higher order terms not taken into account would lower this number by $\approx 10 \%$, leading to a prediction close to 0.8 .

- In the second method, two jet events are selected (soft gluon case) and the azimuthal correlations are computed using the sphericity axis of the event as the reference axis. These we refer to as Two Particle Azimuthal Correlations (TPAC). The pseudorapidity $\eta_{2}$ of the second particle in (3) is taken at a fixed distance from $\eta_{1}$. That is to say, one considers the quantity:

$$
\frac{d C}{d \varphi}\left(\eta_{1}, \eta_{12}, \varphi\right)=\sigma \frac{d \sigma}{d \eta_{1} d \eta_{12} d \varphi} /\left(\frac{d \sigma}{d \eta_{1}} \cdot \frac{d \sigma}{d \eta_{2}}\right)
$$

where $\eta_{12}=\eta_{2}-\eta_{1}$, for any pair of particles.

Section 2 of this paper describes the data selection, section 3 explains how the azimuthal correlations are obtained and the errors are discussed in section 4. Experimental correlations are computed with hadrons and are compared to QCD Monte Carlo model calculations with and without interference effects. The models used in this analysis are presented in section 5 and the comparison with the data is given in section 6 . The interpretation of the results is given in section 7 , while section 8 contains the conclusions.

\section{The OPAL Detector and Data Sample}

This analysis is based on approximately 146,900 multihadronic $\mathrm{Z}^{0}$ decays collected with the OPAL detector at the $\mathrm{e}^{+} \mathrm{e}^{-}$collider LEP corresponding to an integrated luminosity of $6.5 \mathrm{pb}^{-1}$. The OPAL detector is described in detail in reference [9]. The selection of the multihadronic events follows the method described in reference [10].

The detector elements most relevant for this analysis are a large volume central tracking detector and an electromagnetic calorimeter composed of lead glass blocks. The tracking chamber allows an almost $100 \%$ track finding efficiency in the polar angle region $|\cos \theta|<0.92$, where the angle $\theta$ is defined relative to the beam axis. Electromagnetic energy deposits are measured for over $98 \%$ of the solid angle with the calorimeter. Each lead glass block subtends approximately $40 \mathrm{mrad}$ x $40 \mathrm{mrad}$.

Charged tracks were accepted if they originated from within $5 \mathrm{~cm}$ of the interaction point in the plane perpendicular to the beam axis and within $25 \mathrm{~cm}$ in the longitudinal direction. Each charged track was required to have a transverse momentum with respect to the beam direction of more than $150 \mathrm{MeV} / \mathrm{c}$ and at least 40 measured space points. Electromagnetic clusters were accepted if they had over $200 \mathrm{MeV}$ of energy and were spread over at least two lead glass blocks. Only clusters which were not associated with charged tracks were kept [3], and these will be referred to as neutral particles. Hadronic events were required to contain at least five charged tracks which satisfied the above criteria. The polar angle of the thrust axis was required to satisfy $\left|\cos \theta_{\text {thrust }}\right|<0.9$ and the sphericity axis $\left|\cos \theta_{s p h}\right|<0.875$. The total energy of charged particles was required to lie between 20 and $100 \mathrm{GeV}$. About 114,000 multihadronic events were accepted, with an average centre of mass energy of 91.3 $\mathrm{GeV}$. 
For the TPAC study, two-jet events were selected using the E0 recombination scheme for jet reconstruction [11], with a scaled invariant mass parameter $y_{c u t}=0.05$. About 77,500 events remained after applying this cut.

\section{Measurement of the Azimuthal Correlations}

As outlined in the introduction, the EMMC is defined by taking each particle of an event in turn to define an axis, with respect to which the pseudorapidity $\eta$ of the other particles is computed. The azimuthal correlations are calculated if both particles of a pair fall into the same pseudorapidity interval. We calculate the EMMC in the pseudorapidity interval $1<\eta<2$, with $\varphi$ defined in the interval $[0, \pi]$. The pseudorapidity interval is strictly positive as recommended by the authors of [6], in order to reduce the contribution from hard three-jet events. We calculate the TPAC (see formula (4)) in the two intervals $0<\left|\eta_{1}\right|<1$ and $1<\left|\eta_{1}\right|<2$, with $0<\left|\eta_{12}\right|<1$.

The correlation functions are computed using the accepted charged tracks and neutral particles as defined in section 2. The distributions are then corrected bin-by-bin for detector effects and acceptance and for initial state radiation, using a detailed Monte Carlo simulation of the detector [12]. Furthermore, the $\pi^{0}, K_{s}^{0}$ and hyperons are considered to be stable particles at the hadronic level to which the data are corrected. The correction factors were obtained by computing, with the simulated events, the ratio of the correlations obtained with tracks and neutral particles to those obtained at the hadronic level. The corrections were computed using the Jetset parton shower model (version 7.2) [13], with parameters tuned to the OPAL data [14]. A sample of 111,000 Monte Carlo events (after the selection described in section 2) was used for the EMMC study and 75,000 for the TPAC, to calculate the correction factors, which were found to be close to $1 \mathrm{in}$ most of the $\varphi$ region within $10 \%$.

The corrected data for the EMMC are shown in Figure 1a and those for the TPAC are shown in Figures 2a and 2c, for the two pseudorapidity intervals $0<\left|\eta_{1}\right|<1$ and $1<\left|\eta_{1}\right|<2$, respectively.

\section{Discussion of the Errors}

To obtain the statistical errors on the data, the full sample was divided into 10 subsamples of equal numbers of events. The errors were calculated from the mean squared deviation determined for each $\varphi$ bin, on the assumption that the subsamples are normally distributed. These errors were combined quadratically with the statistical errors of the correction coefficients, where the latter were obtained by dividing the full Monte Carlo sample into subsamples in the same way as the data. For the EMMC, the statistical errors are small and less than 0.006 for all $\varphi$. For the TPAC, they are less than 0.015 for all $\varphi$ and for both pseudorapidity configurations.

For the experimental systematic errors, we distinguish errors due to imperfections of the detector simulation and errors due to the choice of the model used for the corrections. The systematics resulting from imperfections of the detector simulation were assessed by comparing the correlations obtained using charged or neutral particles only. We have taken the difference between the corrected distributions to be a measure of the systematic uncertainties in the detector simulation. For the EMMC, these systematic errors are smaller than 0.02 for all $\varphi$, except for $\varphi<0.6$ where they reach 0.10 . For the TPAC, they are about 0.04 on average. 
The systematic errors caused by the selection of a particular model, namely Jetset, for the bin-bybin corrections have been estimated by two different methods. In the first, the data were corrected with new correction coefficients obtained using events generated with the Herwig Monte Carlo, version 5.0 [15], whose parameters were adjusted to the OPAL data. The errors were taken to be the difference between the data corrected by these two models. The average over $\varphi$ for this error is 0.016 for the EMMC and about 0.020 for the TPAC. In the second method, the correction coefficients were obtained using Cojets 6.12 events [18]. This model was chosen because, as discussed in the following sections, it does not describe the observed correlations: it thus provides an important test of the stability of the results to the correction process. In this second evaluation, the systematic error was taken to be the difference between the Jetset and Cojets corrected data; averaged over $\varphi$, it was found to be 0.026 for the EMMC and 0.040 for the TPAC. These values are consistent with those obtained in the evaluation which uses Herwig. The systematic errors are conservatively taken to be the maximum of the two evaluations.

Adding the statistical and systematic errors in quadrature, we obtain, for $\varphi>1$, a mean error of 0.03 for EMMC, and errors of 0.05 and 0.04 for the two pseudorapidity domains considered in the TPAC study. In Figures 1 and 2, the statistical errors are represented as bars, and the domain spanned by the total systematic errors is shown as a grey area. The values of the corrected data with their errors are given in Table 1, where the first error is the statistical one and the second is the total systematic error.

\section{$5 \quad$ Comparison with QCD Monte Carlo programs}

In Monte Carlos without interference effects, the incoherent parton shower is developed according to the Leading Log Approximation (LLA). In order to simulate the effects of coherence, Monte Carlos use approximations such as angular ordering for those based on the LLA, or gluon radiation by colour dipoles.

The QCD Monte Carlo models used in the analysis are the following:

JETSET version $7.2[13]$ has been used to simulate events with and without interference effects. In both cases, the hadronisation follows the Lund string scheme [16]. Interference effects are simulated through polar angle ordering, and it is possible to force an azimuthal asymmetry in the decay of gluons due to colour interference. This last option has also been used and from now on the models will be referred to as J1 (no azimuthal asymmetry) and J2 (with azimuthal asymmetry). The parameters used for J2 are the same as those of J1. Events with incoherent parton showers have also been generated and this model will be denoted J3. ${ }^{1}$

ARIADNE version 3.1 [17], is based on gluon radiation by colour dipoles and hadronisation according to the Lund string scheme. Gluon emission by dipoles allows an almost complete description of interference effects to be included in this Monte Carlo.

COJETS [18] uses an incoherent shower and independent hadronisation. Two versions are used: Cojets 6.12 and Cojets 6.20. They differ only in the simulation of the hadronisation; Cojets 6.20 was tuned in a attempt to fit data on the string effect and quark-gluon jet differences at LEP [19]. In Cojets 6.12 , the quark and gluon jets have the same hadronisation properties.

\footnotetext{
${ }^{1}$ The incoherent parton shower is obtained in Jetset by setting MSTJ $(42)=1$, MSTJ $(44)=1, \operatorname{MSTJ}(46)=0$. The same flags are set to $2,2,0$ for $\mathrm{J} 1$ and to $2,2,2$ for $\mathrm{J} 2$, respectively.
} 
HERWIG version 5.0 [15] generates a parton shower with effective angular ordering and azimuthal asymmetry. The partons are hadronised according to the cluster model.

Models based on matrix elements have not been considered, because soft gluons are absent.

The parameters of these models were adjusted to describe LEP data [14, 19]. For J3, we have determined the main parameters, as well as their errors, by fitting simultaneously the Thrust, the Thrust major, the Sphericity, the Aplanarity, the second Fox-Wolfram moment [14] and the 1/x distributions [2]. We have used the same distributions to determine errors on the main Cojets parameters. The errors were determined by calculating the error matrix from the $\chi^{2}$ difference between the data and the Monte Carlo as model parameters were changed. The parameters used in these models are given in Table 2. The $\chi^{2}$ are comparable to those obtained with the coherent models.

The predictions of the models are shown in Figure 1 for EMMC and in Figure 2 for TPAC, in comparison to the data. The predictions of the incoherent parton shower models are shown as bands. The band widths correspond to a variation in their parameters of plus or minus one standard deviation (see Table 2) and represent the envelope of the predictions.

\section{Discussion of the Results}

In Figure 1a the corrected EMMC data show a strong correlation at $\varphi \approx 0$ which drops below 1 for $\varphi \rightarrow \pi$. At $\varphi=\pi, C(\pi)=0.787 \pm 0.002 \pm 0.04$, where the first error is statistical and the second error is the total systematic uncertainty. This value is significantly above the lowest order calculation $(7 / 16)$, and under the prediction of the recent higher order calculations $(\approx 0.93)$ [8]. The difference between the prediction and the data could be attributed to higher order terms beyond the precision of the calculation as argued in [8]. In Figure 1a, the corrected EMMC data are compared with J1 and J3, Herwig, Ariadne and Cojets 6.20. The global shape of the distribution is reproduced by all models. This is also true for J2 and Cojets 6.12, which are not included in Figure 1a in order to keep the figure simple. The value obtained for Ariadne $(0.786)$ at $\varphi=\pi$ is in good agreement with the measured correlations. For the other models, $C(\pi)$ is slightly higher: about 0.80 for the coherent models (J1, $\mathrm{J} 2$ and Herwig) and roughly 0.85 for the incoherent ones. The qualitative agreement of the model predictions with the shape of the distribution is partly a consequence of the analysis technique itself, which, by taking each particle as an axis in turn, creates correlations at small $\varphi$ and depopulates the region at $\varphi \approx \pi$. To understand this, consider a $q \bar{q}$ event without gluon radiation, which produces two back-to-back jets. For a random set of three hadrons $i, j$ and $k$ for which the particle $i$ is off the $q \bar{q}$ axis, the azimuthal angle between $j$ and $k$ tends to be less than $\pi / 2$. This results in an apparent correlation effect which is not related to coherence.

In order to distinguish more clearly between the models, the difference between model predictions and data is shown in Figure $1 \mathrm{~b}$. The J2 prediction is very similar to that of $\mathrm{J} 1$ and therefore is not shown. Qualitatively, one sees a clear distinction between the incoherent and coherent models, with the latter in better agreement with the data. Amongst the coherent models, Jetset and Ariadne give somewhat smaller correlations than the data at small $\varphi$, while the correlations from Herwig are larger but within systematic errors. For $\varphi \approx \pi$, the agreement is good, except that the predictions of Jetset and Herwig are slightly high, as already mentioned. For incoherent models, the disagreement with the data is much larger. For Jetset (J3) and Cojets 6.20, the correlations are systematically larger than the data by about 0.080 throughout the $\varphi$ interval. Adding in quadrature the statistical and systematic errors, this difference corresponds to two to three standard deviations, for $\varphi>1$. For Cojets 6.12 , the difference with the data varies linearly with $\varphi$, from -0.1 at $\varphi=0$ to become positive and reach $\approx 0.1$ at $\varphi=\pi$. 
Figure 2 shows the corrected TPAC data in comparison with the model predictions. Again, a clear distinction is apparent between the coherent and incoherent models, with the coherent models in considerably better agreement with the data. Amongst the coherent models, and with the parameters used in this analysis, a better description of the data is given by Ariadne than by Jetset (J1 or J2) or Herwig, at least for $\varphi>1$. For the incoherent models, the distributions have a different shape compared to the data, but the same observations as for the EMMC apply. Jetset (J3) and Cojets 6.20 agree with the data for $\varphi \approx 0$, but their correlations are larger than the measured ones for other $\varphi$ values. For $\varphi=\pi$, the model predictions are more than four standard deviations above the data. For models J3 and Cojets 6.20 the differences between the predictions and the data, averaged over $\varphi>1$, are respectively 3.7 and 5.0 standard deviations for the first pseudorapidity interval, and 4.4 and 5.2 for the second interval. For Cojets 6.12, the comparison with the data shows the same trend as for the EMMC.

From these figures, it is seen that Ariadne provides the best description of the data in the region $\varphi \approx \pi$, while the predictions of the incoherent models Cojets 6.20 and J3 are very similar and in strong disagreement with the data. Furthermore, the two versions of Cojets, while predicting very different shapes for the correlation functions, yield a similar prediction for $\varphi \approx \pi$. Models J1 and J3 both use string hadronisation, but the first includes coherence in the parton shower while the second does not. The fact that $\mathrm{J} 3$ does not reproduce the distributions implies that hadronisation alone is not responsible for the measured effects. For Jetset, with the parameters used, the mean parton multiplicity is large, perhaps making the details of the hadronisation model less important for these distributions. Thus we conclude that hadronisation effects have an important influence on the general shape of the distributions, but that the correlations observed for $\varphi \approx \pi$ are sensitive to the presence of coherence in the parton shower. The relative abilities of the different parton shower models which include coherence to describe the data are similar to those observed in the recent OPAL study of two-particle momentum correlations [20]: in particular, Ariadne provides the best description of the data.

For small $\varphi$, it is possible that the azimuthal correlations are influenced by Bose-Einstein effects. Using the possibility in Jetset to switch Bose-Einstein effects on or off, and the parameters determined by the OPAL Collaboration [21], we have checked that these effects are negligible at the hadron level. We have also studied the variation of the TPAC when $y_{\text {cut }}$ is changed from 0.05 to 0.03 . The differences between the $\mathrm{J} 1$ and $\mathrm{J} 3$ models are observed to persist, although at a slightly reduced level. Finally, we have tested the stability of the TPAC as the particle multiplicity changes. This has been done using model J1, keeping its parameters fixed, and varying the multiplicity by eliminating some particles at random. The TPAC are stable to within $0.1 \%$ when the multiplicity is reduced by $20 \%$.

The two azimuthal correlation distributions, EMMC and TPAC, yield consistent conclusions, although the TPAC is observed to be more sensitive to the differences between models for $\varphi>1$.

\section{$7 \quad$ Interpretation of the Results}

In this section, we study using Monte Carlos how the correlations computed with partons compare with the theoretical expectation discussed in the introduction (Equations 1 and 2). Secondly, we examine the connection between the hadronic and the partonic distributions. These studies allow us to make a better assessment of how the measured distributions are related to parton coherence. 


\subsection{The partonic correlations}

The Two Particle Azimuthal Correlations calculated with partons for different Monte Carlo models are shown in Figure 3. The J3 distribution is clearly flatter than the distributions for the models which include interference effects. These distributions are strongly distorted in the region $\varphi<1$ by the non-perturbative cutoff used in the models. For coherent showers, this cutoff acts to inhibit small values of relative transverse momenta between partons, and therefore suppresses small angles between them. This effect is clearly visible in, for example, the J1 curves in Figures 3 . The distributions are also distorted in the region $\varphi \approx \pi$ by correlations produced by the conservation of momentum. By means of an example we will illustrate this correlation. Let us consider a three parton $q_{1} q_{2} g_{3}$ configuration, in which the transverse momentum with respect to the event axis is mainly balanced between $g_{3}$ and say $q_{2}$. In the plane perpendicular to the event axis the particles produced by $q_{2}$ tends to be back to back with the particles from $g_{3}$. Therefore, this effect produces a correlation at $\varphi \approx \pi$ which is particularly visible in Figures $3 \mathrm{c}$ and $3 \mathrm{~d}$. Now let us consider the addition of a softer gluon $g_{4}$ to the event. The effects of QCD coherence are expected to manifest themselves in the correlations between particles produced by $g_{3}$ and $g_{4}$. These particles will generally have lower pseudorapidities than those produced by $q_{2}$. It therefore seems natural to expect that the correlation at $\varphi \approx \pi$ from momentum conservation will be most apparent in the higher pseudorapidity interval $1<\left|\eta_{1}\right|<2$.

The above discussion illustrates that the kinematical interval $0<\left|\eta_{1}\right|<1$, with $\varphi>\pi / 2$, is less affected than are the other regions and that it is the region most sensitive to coherence effects. Figures $3 \mathrm{a}$ and $3 \mathrm{~b}$ show that, in this region, the models with coherence demonstrate the expected behaviour, i.e. they lie below the prediction of the incoherent model J3 for $\varphi>\pi / 2$. The distributions for Cojets are not shown because the average number of partons in this model is small $(\approx 3.25)$, due to its large value for the non-perturbative cutoff, and consequently the distortion due to momentum conservation is very large.

Similarly for the EMMC, the distributions lie below 1 for all the models with coherence for $\varphi>\pi / 2$, falling to a value of about 0.8 for $\varphi \approx \pi$, while the J3 distribution is flatter and stays above 1 , as is seen in Figure 4a. For $\varphi \rightarrow \pi$, the models with interference therefore behave as expected from Equation 1, although the correlations introduced by the analysis technique and described in Section 6 also play a role.

\subsection{The role of hadronisation}

We next compare the TPAC for partons (Figure 3 ) with those for hadrons (Figure 2) in the kinematical region $0<\left|\eta_{1}\right|\langle 1, \varphi\rangle \pi / 2$, which was shown above to be the most sensitive to the underlying QCD process. In this region, the parton and hadron level distributions maintain their relative positions for the J1, J2, J3, Herwig and Ariadne models, with the exception that J2 (not shown in Figure 2) lies above Ariadne in the hadron distribution but below it for partons. This implies that hadronisation does not greatly affect the coherence signal observed at the parton level in this kinematical region.

The two versions of Cojets have the same parton shower development and differ only in the hadronisation; therefore, the different shapes of the hadronic distributions for these two models seem to indicate that hadronisation causes strong distortions of the parton distribution. However, restricting ourselves to the domain $0<\left|\eta_{1}\right|<1, \varphi \approx \pi$, we see that the predictions of the two models are roughly the same after hadronisation, indicating again that this region gives a good picture of the underlying parton structure.

One surprising result is that the hadronic TPAC distributions of $\mathrm{J} 1$ and $\mathrm{J} 2$ are very close, while 
the corresponding parton distributions are different. These two models use the same hadronisation parameters. The consistency between the azimuthal distributions for J1 and J2 at the hadron level may reflect the non-local nature of string hadronisation: the hadronic distributions depend on all the characteristics of the underlying event which are sensitive to coherence phenomena, and not just the azimuthal properties of the partons. From this discussion we conclude that the azimuthal correlations observed at the hadron level are sensitive to the global parton topology of the events, but not to its details.

The same discussion can also be applied to the EMMC. In order for a comparison with model predictions to be meaningful, the Monte Carlo parton cascade must develop sufficiently far to be sensitive to coherence phenomena. For those models which fulfil this condition, namely all the coherent models and the incoherent model J3, the hadronisation corrections are small in the region $\varphi \approx \pi$ and the shapes of the correlation curves are comparable at the parton and hadron levels for $\varphi>\pi / 2$. We thus infer that these distributions give a reasonable description of the underlying parton structure.

\section{Conclusion}

We have examined phenomena related to coherence in multi-gluon radiation by studying azimuthal correlations between particles produced in $\mathrm{Z}^{0}$ hadronic decay events. In this paper, we have used two methods. The first employs the Energy-Multiplicity-Multiplicity Correlations distribution proposed by Dokshitzer et al., which does not require any special event selection. The second method employs Two Particle Azimuthal Correlations in selected two-jet events. Both methods lead to the same conclusions, although the second is found to be somewhat more sensitive.

We find this study of correlations to be a useful tool to discriminate between the soft gluon modelling as incorporated into different QCD Monte Carlo programs. The correlations clearly favour models which include interference effects in the parton shower development, despite the fact that all the models provide a good description of the global properties of $\mathrm{Z}^{0}$ hadronic events. The comparison of Jetset with an incoherent parton shower to models which include interference effects implies that string hadronisation alone is not able to reproduce the observed correlations: therefore, coherence is relevant to the measured distributions. The interpretation of the data in terms of the QCD analytic formula is complicated by hadronisation effects, which distort the underlying distributions, especially for $\varphi<\pi / 2$, and by kinematical effects near $\varphi=\pi$. Despite these complications, it is possible to state that for $\varphi>\pi / 2$, the observed correlations reflect the analytic QCD expectations.

\section{Acknowledgements}

It is a pleasure to thank the SL Division for the efficient operation of the LEP accelerator, the precise information on the absolute energy, and their continuing close cooperation with our experimental group. In addition to the support staff at our own institutions we are pleased to acknowledge the Department of Energy, USA, National Science Foundation, USA, Science and Engineering Research Council, UK, Natural Sciences and Engineering Research Council, Canada, Israeli Ministry of Science, Minerva Gesellschaft, Japanese Ministry of Education, Science and Culture (the Monbusho) and a grant under the Monbusho International Science Research Program, American Israeli 
Bi-national Science Foundation, Direction des Sciences de la Matière du Commissariat à l'Energie Atomique, France, Bundesministerium für Forschung und Technologie, FRG, National Research Council of Canada, Canada, A.P. Sloan Foundation and Junta Nacional de Investigação Científica e Tecnológica, Portugal.

\section{References}

[1] A. Bassetto, M. Ciafaloni, G. Marchesini, Phys. Rep. 100 (1983) 201 ; Y.L. Dokshitzer et al., Rev. Mod. Phys. 60 (1988) 373.

[2] OPAL Coll., M.Z. Akrawy et al., Phys. Lett. B247 (1990) 617;

L3 Coll., B. Adeva et al., Phys. Lett. B259 (1991) 199.

[3] OPAL Coll., M.Z. Akrawy et al., Phys. Lett. B261 (1991) 334.

[4] JADE Coll., W. Bartel et al., Phys. Lett. B101 (1981) 129;

W. Bartel et al. Z. Phys. C21 (1983) 37.

[5] Y.I. Azimov et al., Phys. Lett. B165 (1985) 147.

[6] Y.L. Dokshitzer et al., Phys. Lett. B245 (1990) 243.

[7] D. Amati and G. Veneziano, Phys. Lett. B83 (1979) 87;

Y.I. Azimov et al., Z. Phys. C27 (1985) 65.

[8] Y.L. Dokshitzer, G. Marchesini and G. Oriani, Lund preprint LUTP 92/19 (1992).

[9] OPAL Coll., K. Ahmet et al., Nucl. Inst. and Meth. A305 (1991) 275.

[10] OPAL Coll., P.D. Acton et al., Z. Phys. C55 (1992) 1.

[11] OPAL Coll., M.Z. Akrawy et al., Z. Phys. C49 (1991) 375.

[12] OPAL Coll., J. Allison et al., Nucl. Inst. and Meth. A317 (1992) 47.

[13] T. Sjöstrand, Comp. Phys. Comm. 39 (1986) 347;

T. Sjöstrand and M. Bengtsson, Comp. Phys. Comm. 43 (1987) 367.

[14] OPAL Coll., M.Z. Akrawy et al., Z. Phys. C47 (1990) 505.

[15] G. Marchesini and B.R. Webber, Nucl. Phys. B310 (1988) 461.

[16] B. Andersson et al., Phys. Rep. 97 (1983) 31.

[17] L. Lönnblad, LUTP 91/11; Comp. Phys. Comm. 59 (1990) 527.

[18] R. Odorico, Comp. Phys. Comm. 32 (1984) 139.

[19] P. Mazzanti and R. Odorico, Nucl. Phys. B370 (1992) 23;

P. Mazzanti and R. Odorico, University of Bologna preprint DFUB 92/1 (1992).

[20] OPAL Coll., P.D. Acton et al., Phys. Lett. B287 (1992) 401.

[21] OPAL Coll., P.D. Acton et al., Phys. Lett. B267 (1991) 143. 
Table 1. Corrected data for EMMC and TPAC

The first error is statistical and the second is the total systematic error.

\begin{tabular}{|c|c|c|c|}
\hline \multirow{2}{*}{$\begin{array}{c}\varphi \\
\text { (radian) }\end{array}$} & & \multicolumn{2}{|c|}{ TPAC } \\
\hline & $1<\eta<2$ & $0<\left|\eta_{1}\right|<1$ & $1<\left|\eta_{1}\right|<2$ \\
\hline-0.0982 & $3.126 \pm 0.006 \pm 0.126$ & $5 \pm 0.367$ & 1.66 \\
\hline $0.0982-0.1964$ & $2.923 \pm 0.006 \pm 0.111$ & $1.665 \pm 0.012 \pm 0.165$ & $1.653 \pm$ \\
\hline $0.1964-0.2945$ & $2.646 \pm 0.005 \pm 0.096$ & $1.614 \pm 0.014 \pm 0.085$ & $1.595 \pm 0$ \\
\hline $0.2945-0.3927$ & $2.385 \pm 0.005 \pm 0.082$ & $1.558 \pm 0.010 \pm 0.100$ & $1.561 \pm 0.008 \pm 0.050$ \\
\hline $0.3927-0.4909$ & $2.155 \pm 0.005 \pm 0$ & $1.498 \pm 0.013 \pm 0.105$ & $1.506 \pm$ \\
\hline $0.4909-0.5891$ & $1.960 \pm 0.004 \pm 0$ & $1.485 \pm 0.011 \pm 0.090$ & $1.453 \pm 0.006 \pm 0.040$ \\
\hline $0.5891-0.6872$ & $1.793 \pm 0.004 \pm$ & $1.436 \pm 0.012 \pm 0.073$ & $1.407 \pm 0.007 \pm 0.033$ \\
\hline $0.6872-0.7854$ & $1.653 \pm 0.0$ & $10 \pm 0.065$ & $1.364 \pm$ \\
\hline $0.7854-0$. & $1.531 \pm 0$. & $1 \pm 0.063$ & $1.326 \pm$ \\
\hline $0.8836-0.9818$ & $1.431 \pm 0.0$ & $11 \pm 0.062$ & $1.280 \pm$ \\
\hline $0.9818-1.0799$ & $1.344 \pm 0.0$ & $1.281 \pm 0.010 \pm 0.062$ & $1.251 \pm 0.007 \pm 0.027$ \\
\hline $1.0799-1.1781$ & $1.265 \pm 0.0$ & $9 \pm 0.058$ & $1.215 \pm 0$ \\
\hline $1.1781-1.2763$ & $1.202 \pm 0$ & $1.222 \pm$ & $1.193 \pm$ \\
\hline $1.2763-1.3745$ & $1.143 \pm 0$ & $1.158 \pm$ & $1.169 \pm$ \\
\hline 4726 & $1.093 \pm 0$ & $1.159 \pm$ & $1.155=$ \\
\hline $1.4726-1.5708$ & $1.047 \pm 0.003 \pm$ & $1.120 \pm 0.012 \pm 0.033$ & $1.144 \pm 0.006 \pm 0.027$ \\
\hline $08-1.6690$ & $1.007 \pm 0$ & $1 \pm 0.035$ & $1.133 \pm$ \\
\hline $1.6690-1.7672$ & $0.971 \pm 0.0$ & $8 \pm 0.041$ & $1.132 \pm$ \\
\hline $1.7672-1.8653$ & $0.945 \pm 0.0$ & $0 \pm 0.048$ & $1.140 \pm$ \\
\hline $1.8653-1.9635$ & $0.921 \pm 0.0$ & $1 \pm 0.053$ & $1.147 \pm 0.006 \pm 0.036$ \\
\hline $1.9635-2.0617$ & $0.893 \pm 0.003 \pm 0.030$ & $1.055 \pm 0.008 \pm 0.055$ & $1.142 \pm 0.006 \pm 0.037$ \\
\hline $2.0617-2.1599$ & $0.876 \pm 0.0$ & $011 \pm 0.049$ & $1.143 \pm 0.008 \pm 0.035$ \\
\hline $2.1599-2.2580$ & $0.855 \pm 0.002 \pm c$ & $1.018 \pm 0.011 \pm 0.038$ & $1.169 \pm 0.006 \pm 0.030$ \\
\hline $2.2580-2.3562$ & $0.836 \pm 0.002 \pm 0$ & $1.004 \pm 0.006 \pm 0.035$ & $1.175 \pm 0.006 \pm 0.029$ \\
\hline $2.3562-2.4544$ & $0.829 \pm 0.002 \pm 0.034$ & $1.000 \pm 0.010 \pm 0.044$ & $1.201 \pm 0.005 \pm 0.032$ \\
\hline $2.4544-2.5526$ & $0.816 \pm 0.002 \pm 0.035$ & $0.994 \pm 0.006 \pm 0.051$ & $1.222 \pm 0.006 \pm 0.035$ \\
\hline $2.5526-2.6507$ & $0.807 \pm 0.003 \pm$ & $11 \pm 0.050$ & $1.249 \pm 0.006 \pm 0.034$ \\
\hline $2.6507-2.7489$ & $0.798 \pm 0.003 \pm 0.1$ & $0.983 \pm 0.010 \pm 0.047$ & $1.272 \pm 0.006 \pm 0.033$ \\
\hline $2.7489-2.8471$ & $0.793 \pm 0.002 \pm 0.033$ & $0.994 \pm 0.008 \pm 0.053$ & $1.298 \pm 0.008 \pm 0.042$ \\
\hline $2.8471-2.9453$ & $0.786 \pm 0.003 \pm 0.033$ & $0.993 \pm 0.010 \pm 0.068$ & $1.317 \pm 0.006 \pm 0.059$ \\
\hline 2 & $0.785 \pm 0$ & $0.979 \pm$ & $1.330 \pm 0.007 \pm 0.059$ \\
\hline $3.0434-3.1416$ & $0.787 \pm 0.002 \pm 0.039$ & $0.975 \pm 0.011 \pm 0.060$ & $1.346 \pm 0.007 \pm 0.037$ \\
\hline
\end{tabular}


Table 2. QCD Monte Carlo parameters.

Only the errors calculated in this study are given.

\begin{tabular}{|c|c|c|c|}
\hline Model & Parameter & MC name & Value \\
\hline \multirow[t]{5}{*}{ Jetset 7.2 coherent $\mathrm{J} 1, \mathrm{~J} 2$} & $\Lambda(\mathrm{GeV})$ & PARJ(81) & 0.29 \\
\hline & $Q_{0}(\mathrm{GeV})$ & PARJ(82) & 1.0 \\
\hline & $\sigma_{q}(\mathrm{GeV})$ & PARJ(21) & 0.37 \\
\hline & & PARJ(41) & 0.18 \\
\hline & $b\left(\mathrm{GeV}^{-2}\right)$ & PARJ $(42)$ & 0.34 \\
\hline \multirow[t]{5}{*}{ 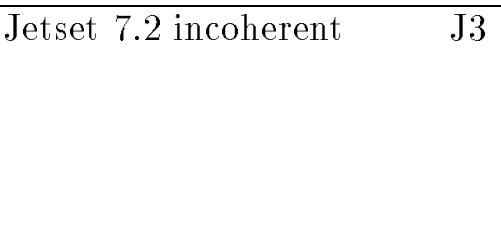 } & $\Lambda(\mathrm{GeV})$ & PARJ(81) & $0.44 \pm 0.06$ \\
\hline & $Q_{0}(\mathrm{GeV})$ & $\operatorname{PARJ}(82)$ & 1.45 \\
\hline & $\sigma_{q}(\mathrm{GeV})$ & PARJ(21) & $0.44 \pm 0.03$ \\
\hline & & PARJ(41) & 0.18 \\
\hline & $b\left(\mathrm{GeV}^{-2}\right)$ & PARJ(42) & $0.65 \pm 0.15$ \\
\hline \multirow[t]{5}{*}{ Ariadne 3.1} & $\Lambda(\mathrm{GeV})$ & $\operatorname{VAR}(1)$ & 0.25 \\
\hline & $P_{T} \min (\mathrm{GeV})$ & $\operatorname{VAR}(3)$ & 0.50 \\
\hline & $\sigma_{q}(\mathrm{GeV})$ & PARJ $(21)$ & 0.35 \\
\hline & & PARJ(41) & 0.50 \\
\hline & $b\left(\mathrm{GeV}^{-2}\right)$ & $\operatorname{PARJ}(42)$ & 0.75 \\
\hline \multirow[t]{4}{*}{ Cojets 6.12} & $\Lambda(\mathrm{GeV})$ & ALAMBD & $0.17 \pm 0.030$ \\
\hline & $Q_{0}(\mathrm{GeV})$ & CUTJET & 3.0 \\
\hline & $\sigma_{q}(\mathrm{GeV})$ & SIGMAQ & $0.52 \pm 0.024$ \\
\hline & $\sigma_{g}(\mathrm{GeV})$ & SIGMAG & $0.52 \pm 0.024$ \\
\hline \multirow[t]{4}{*}{ Cojets 6.20} & $\Lambda(\mathrm{GeV})$ & ALAMBD & $0.17 \pm 0.030$ \\
\hline & $Q_{0}(\mathrm{GeV})$ & CUTJET & 3.0 \\
\hline & $\sigma_{q}(\mathrm{GeV})$ & SIGMAQ & $0.85 \pm 0.045$ \\
\hline & $\sigma_{g}(\mathrm{GeV})$ & SIGMAG & $0.85 \pm 0.045$ \\
\hline \multirow[t]{5}{*}{ Herwig 5.0} & $\Lambda(\mathrm{GeV})$ & QCDLAM & 0.20 \\
\hline & $M_{g}(\mathrm{GeV})$ & RMASS(13) & 0.75 \\
\hline & $M_{\max }(\mathrm{GeV})$ & CLMAX & 3.50 \\
\hline & $Q_{0}(q)(\mathrm{GeV})$ & VQCUT & 0.48 \\
\hline & $Q_{0}(g)(\mathrm{GeV})$ & VGCUT & 0.06 \\
\hline
\end{tabular}




\section{Figures}

Figure 1: The unfolded EMMC data corrected by Jetset, together with model predictions: full distributions (a) and differences between the models and the data (b). The error bars shown include the statistical errors on the data combined with those of the correction coefficients. The total systematic errors are represented by the grey area. For the incoherent parton shower models, the bands correspond to a variation of the main parameters by plus or minus one standard deviation. The coherent Jetset model $\mathrm{J} 2$ is not shown because its prediction is close to $\mathrm{J} 1$.

Figure 2: The unfolded measurements of the Two Particle Azimuthal Correlations (TPAC), for charged and neutral particles, corrected by Jetset. The errors are presented in the same manner as in Figure 1. Model J2 is not shown because it is close to J1.

Figure 3: The Two Particle Azimuthal Correlations for partons.

Figure 4: The Energy-Multiplicity-Multiplicity Correlations for partons. 